\title{
FLIGHT DECK AUTOMATION SUPPORT WITH DYNAMIC 4D TRAJECTORY MANAGEMENT FOR ACAS: AUTOFLY-AID
}

\author{
Emre Koyuncu*, Eduardo Garcia', Gokhan Inalhan ${ }^{*}$ \\ *Istanbul Technical University, Faculty of Aeronautics and Astronautics, Istanbul, Turkey \\ ${ }^{\dagger}$ CRIDA and Polytechnic University of Madrid, Air Navigation Department, Madrid, Spain
}

\section{Abstract}

AUTOFLY-Aid Project aims to develop and demonstrate novel automation support algorithms and tools to the flight crew for flight critical collision avoidance using "dynamic 4D trajectory management". The automation support system is envisioned to improve the primary shortcomings of TCAS, and to aid the pilot through add-on avionics/head-up displays and reality augmentation devices in dynamically evolving collision avoidance scenarios. The main theoretical innovative and novel concepts to be developed by AUTOFLY-Aid project are a) design and development of the mathematical models of the full composite airspace picture from the flight deck's perspective, as seen/measured/informed by the aircraft flying in SESAR 2020, b) design and development of a dynamic trajectory planning algorithm that can generate at real-time (on the order of seconds) flyable (i.e. dynamically and performance-wise feasible) alternative trajectories across the evolving stochastic composite airspace picture (which includes new conflicts, blunder risks, terrain and weather limitations) and c) development and testing of the Collision Avoidance Automation Support System on a Boeing 737 NG FNPT II Flight Simulator with synthetic vision and reality augmentation while providing the flight crew with quantified and visual understanding of collision risks in terms of time and directions and countermeasures.

\section{Introduction}

Airborne Collision Avoidance Systems and their current implementations such as TCAS are based on infrastructure and operations of ATM realm of the 20th Century. Specifically, in mid 1990s Traffic Alert and Collision Avoidance System (TCAS) [1,2] was introduced to prevent mid-air collisions between aircraft. TCAS I, which is intended primarily for general aviation aircraft, provides traffic advisories (TA) to pilots. In TCAS II, in addition to TA, resolution advisories (RA) are introduced to instruct pilots on how to resolve conflict situations.

In 2008, EUROCAE and RTCA have jointly revised operational standards of TCAS II, which is known as TCAS II version 7.1, to solve some safety issues [3] that caused mid-air collisions. Even with current improvements, the primary shortcomings of TCAS can be summarized under 4 main themes. Specifically,

- TCAS is limited to support vertical separation advisories and the collision avoidance is limited to myopic 1-1 separation strategy. Both of these can result in potential new collision scenarios with other aircraft even though the pilot follows the advisory.

- TCAS dynamic re-routing/re-advisory capability is limited to resolution advisory reversals, and in face of series of pilot blunders this limits the reliability of generated de-conflicts.

- TCAS does not carry weather, terrain, and ground and obstacle awareness and can potentially create advisories resulting in harming scenarios especially in close ground/terrain operation phases.

- TCAS does not provide resolution advisories in line with the aircraft's "current" performance capability and flight envelope limitations. Thus, the system can potentially provide advisors out of the capability of the air vehicle.

Even in that sense, the system has resulted in accident scenarios [6] rather these be from blunders or from the way the information is conveyed to the pilot. With SESAR and its technology developments [4], Airborne Collision Avoidance System (ACAS) implementations can now rely on new 
Communication Navigation Surveillance (CNS) services, trajectory based operations and System Wide Information Management (SWIM) capabilities a) to improve on the short-comings of the existing collision avoidance systems and b) to meet the growing demanding needs of collision avoidance in the face of increasing flight and aircraft capacities [5]. For example, RTCA is further considering several sensor integrations for supporting collision avoidance, including Automatic Dependent Surveillance Broadcast (ADS-B) $[7,8]$ as to enable TCAS for new capabilities such as lateral and speed based avoidance, improved surveillance and tracking systems. In addition, NextGen is currently investigating more delegation of traffic separation responsibility to the pilot $[9,10]$. In the system, pilots are assisted in predicting and resolving loss of separation by cockpit automation, known generally as Airborne Separation Assistance Systems (ASAS) [11, 12]. Early ASAS experiments showed promising results of assisted separation operations [13, 14].

With the ever growing airspace capacities of the Flight plan 2020 and the 2050 vision, SESAR Program Master Plan [4] and the reshaping of the airspace [5] (with user preferred routing, non-segregated flight, new separation modes), for to further minimize the current shortcomings of the TCAS, the airborne collision avoidance need to be supplemented with automation support systems that;

- Enhance the pilot situational awareness by not only utilizing the new SESAR CNS and SWIM infrastructure but also using (and blending with) the on-board avionics that provide weather, ground/terrain and obstacle information,

- Provide alternative de-conflict routes in the event of performance and potential hazard limitations,

- Provide dynamic trajectory planning in the event of new conflicts and potential blunders,

- Enhance system robustness by modeling and taking into account uncertainty associated with data source errors/ failures and pilots' intents, and utilize uncertainty and its time propagation in dynamic trajectory planning,
- Provide the pilot with quantified and visual understanding of collision risks in terms of time and directions and countermeasures.

Towards these goals, AUTOFLY-Aid will study "dynamic 4D trajectory management" to be implemented above the basic/passive TCAS solution (rather the TCAS solution be the standard vertical separation advisory or a variant/improvement on that) using the on-board avionics and the SESAR enhanced flight deck situational awareness (Figure 1), coming from CNS (primarily ADS-B and its enhancements) and SWIM network.

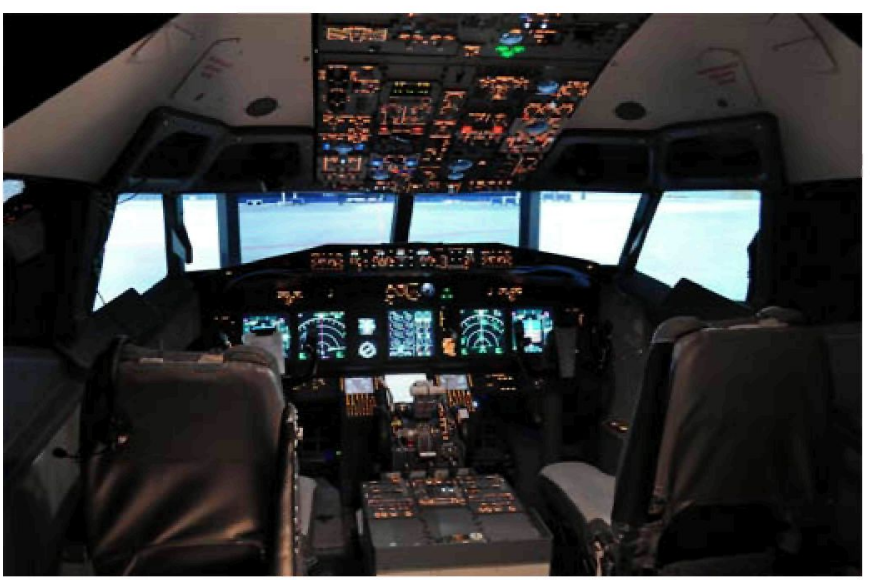

Figure 1. The New B 737 - NG Simulator Replica. Identical System Is Being Built at ITU for Flight Deck ATC Research - Courtesy of Flight Deck Solutions

The "dynamic 4D trajectory management" is to be based on a hybrid and stochastic airspace model not only representing uncertainties associated with sensed and received airspace traffic and intent information, but also representing limitations associated with weather, terrain/obstacle and new conflict hazards. As an end result, the overall automation support system which embeds "dynamic 4D trajectory management" is envisioned to a) provide the pilots with alternative trajectories as tunnels-in-the-sky through avionics displays on the console and head-up displays in real-time, b) provide the flight crew with quantified and visual understanding of collision risks in terms of time and directions and countermeasures, and c) provide autonomous conflict resolution as an autopilot mode. Thus, ensuring highly responsive and adaptive 
airborne collision avoidance in face of ever challenging scenarios that involve blunders, weather/ terrain/ obstacle/ new conflict hazards.

\section{AUTOFLY-Aid Project}

"Real World" factors such as uncertainty in sensing, information, intent and rationality, asynchronous data and information flow with delays, equipment malfunctions, lack of centralized decisionmaking in short to immediate term collision avoidance, make responsive and adaptive airborne collision avoidance challenging. The problem is further complicated by the fact that the process is governed by humans and real aircraft dynamics (and thus with limitations of an aircraft and a human). In addition weather, terrain/ground and obstacle hazards, and new conflicts appearing in dynamically evolving scenarios lead to a potentially unbounded Airborne Collision Avoidance (ACA) problem complexity. However, with assumptions and simplifications, the ACA problem has been studied in depth not only on the fundamental collision detection and avoidance algorithmic perspective, but also on system modeling, systems enhancements, pilot guidance with decision-aiding and automation frontiers. A recent of survey of these efforts can be found in $[15,16]$. Rather the algorithmic efforts hinge on potential fields, geometric and MILP optimization, sampling-based motion planning, policy search or evolutionary methods, the set of underlying assumptions and the algorithmic limitations lead to one or a set of shortcomings such as;

- Kinematically feasible but dynamically infeasible maneuver generation (mainly because of car-like representations of the aircraft),

- Inability to account or model irrationality (seen as a result of blunders),

- Overly conservative (for almost all minmax scenarios there is no safe solution) problem setting leading to illogical solutions (i.e. aircrafts chasing each other),

- Limitations to 2D maneuvering,

- Inability to be implemented at real time because of computational burden,

- Requirement for central processing (rather this be a complete one center solution or a distributed solution that requires central synchronization and updates),

- Requirement for precise synchronization across the maneuvers, and the need for additional (in some cases unrealistic) operational capabilities needing extra Xlinks and navigation devices,

- Inability to account for (or no consideration of) weather, terrain/ground and obstacle patterns, and

- Inability account for "Real World" factors.

In this perspective even the Traffic Alert and Collision Avoidance System (TCAS) can be classified as a fixed-set policy algorithm with intermittent updates that provide synchronized resolution advisories and carry at least three major limitations from the set noted above. It should also be noted that the majority of existing work is more applicable towards mid and mid to short term collision avoidance (most probably as automation or decision support systems at the ATC operator level) rather than airborne short-to-immediate collision avoidance. In the AUTOFLY-Aid project, it is aimed to further improve a set of these limitations and the current state of art in real-time airborne short-toimmediate collision avoidance by focusing on the following 4 main topics.

\section{Dynamic Modeling of the Air Space with Uncertainties}

The dynamic modeling of the Air Space hinges on hybrid systems methodology which provides the framework for not only continuous dynamics but also discrete dynamics and logical jumps (and decisions). With the inclusion of stochastic processes and distributions, we aim to model sensors, devices, information, intent, decisions and aircraft each with uncertainties and discrete/logical element under a coherent systems model. With regards to the representing aircraft dynamics, an in house developed Mode Based Maneuver Automaton is envisioned to serve as the basic starting model. This finite state automaton can not only represent the full dynamics and the limitations of the aircraft but also describe almost any maneuver (excluding voluntary side-slip flight) by maneuver mode sequences. In [17], using this underlying model, a real-time dynamically 
feasible trajectory-planning algorithm is developed using trajectory envelope search approach for highly maneuverable aerial vehicles flying in the 3D complex environments. The main practicality of this approach is in reducing the complexity of both the conflict detection and trajectory-planning phase. Further extension of this model with stochastic elements is to be studied. In addition, other aircraft's intent is to be modeled through a stochastic risk based decision model, which inherently captures all potential blunders and even irrational behavior. The underlying working principle of this decision model and its effect on the collision avoidance is further elaborated in the corresponding subsection. Besides geometric based localization of terrain/ground and obstacles, measurement/information uncertainty and weather pattern is to be modeled through generic (and existing) stochastic sensor/information models and dynamic weather models respectively. In addition, existing ATC operations, directives, sectors and the underlying navigation, guidance and control within the flight deck are to be included in the simulations and automation support system tests.

\section{Dynamic Data and Information Fusion}

Information filters and their extensions to underlying hybrid system dynamics will be studied for dynamic data and information fusions. Specifically, asynchronous updates of data, information and intent will be integrated to the continuous dynamic propagation of underlying hybrid system models. In that extent, initial work and efforts has done on fusing delayed and imperfect information with hybrid system dynamic model (based on Maneuver Based Automaton) for generating dynamically feasible flight strategy in complex environments. Information delays, uncertainties and asynchronies can be handled through the Information Filters. It is expected that this would be further expanded to the AUTOFLYAid's general Air Space realm.

\section{Probabilistic Real-Time Conflict Detection with Uncertainties}

The conflict detection methodology is based on the idea of spatial search phenomena for potential conflicts including aircraft-to-aircraft conflicts (seen in Figure 2) and collisions with the obstacles (rather these obstacles are "soft" weather hazards or "hard" earth objects). This search method to be investigated will rely on creation of probabilistic flight trajectory (4DT) envelopes for the aircrafts in the traffic for every predefined time window. These envelopes also include uncertainty factors existing in weather patterns and the flight models. The flight models naturally embed the stochastic nature in which the rationality (or irrationality) of the flight crews within the common airspace is presented with probabilistic action patterns. Trajectory envelope search process hinges on using multi-modal approach utilizing distinct flight modes. These flight modes can be combined to generate maneuvers within the flight envelope of the aircraft.

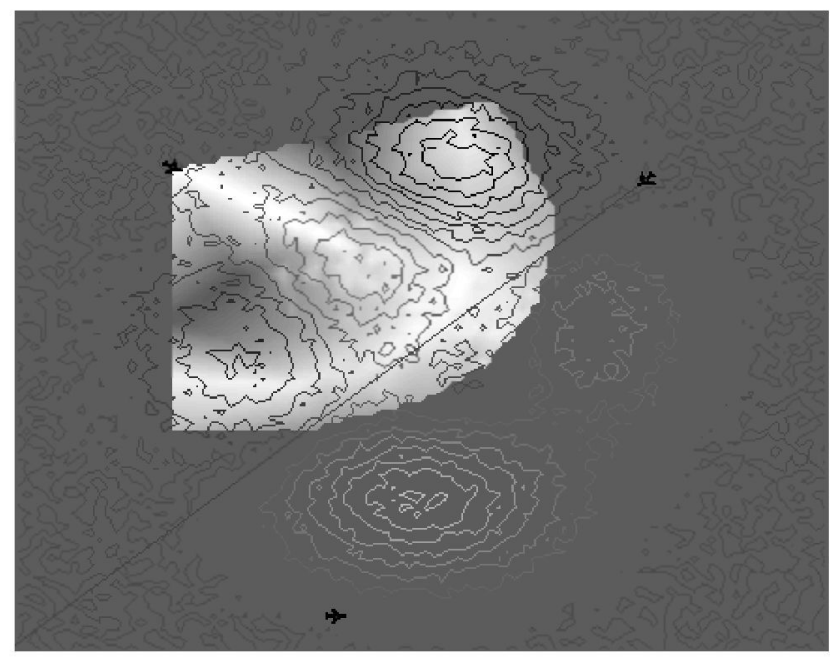

\section{Figure 2. Potential Air Conflict Detection with Trajectory Distribution Maps}

The main idea behind the Modal Maneuver Based PRM (Probabilistic Road Mapping) Planning [18] is to divide an arbitrary flight maneuver into smaller maneuver segments (called maneuver modes) and associate them with maneuver parameters (called modal inputs). The multi-modal maneuver search relies on a finite state automaton, which chooses maneuvers from finite maneuver set and then chooses their parameters from continuous dynamically feasible region. This selection is made randomly in order to cover whole flight envelope, but it is important to assign probability rates to the selections (in case of the lack of knowledge on the flight intents) based on the history of the flight path. Changes in flight path parameters (velocity, acceleration, turn radius, climb/descent-rate) and occurrences can be modeled with stochastic 
processes and distributions. Trajectory distribution map (seen in Figure 3), which is the set of the generated maneuvers in a probabilistic distribution, represents all potential positions of the aircrafts in the future. If the generated 4D trajectory distribution maps outline conflicts at high likelihood rates, this will serves as the alert for potential collision in a predefined unit time. AUTOFLY-Aid's main aim would be to further expand the methodology to create these 4D trajectory distribution maps for the composite Air Space picture (which includes other aircraft, weather patterns, terrain/ground and obstacles) at real-time (on the order of seconds) and identify/classify collision risks and potential additional risk factors. Methodology in conflict detection and avoidance is illustrated in Figure 4.

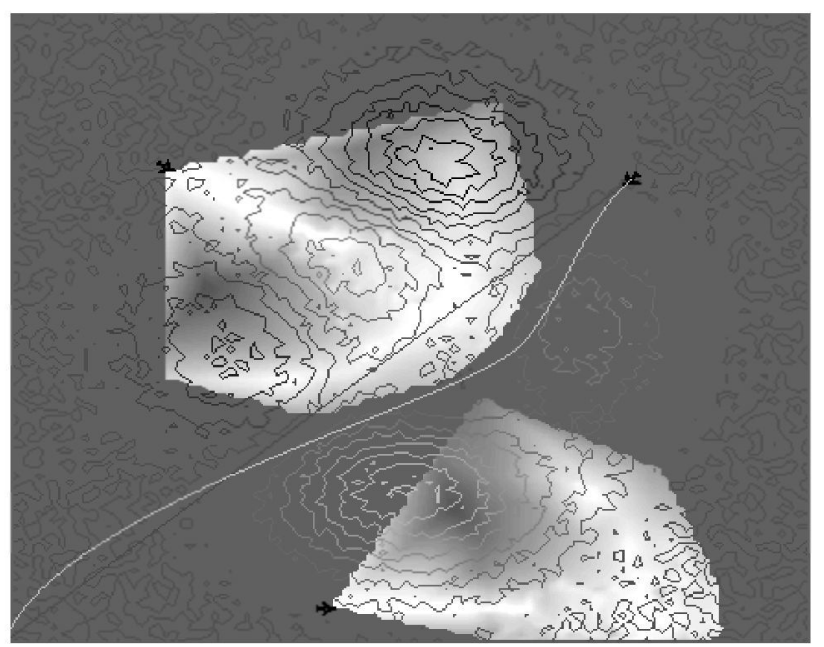

Figure 3. Result of Dynamically Feasible Trajectory Generation in Air Congestion Including Severe Weather Condition

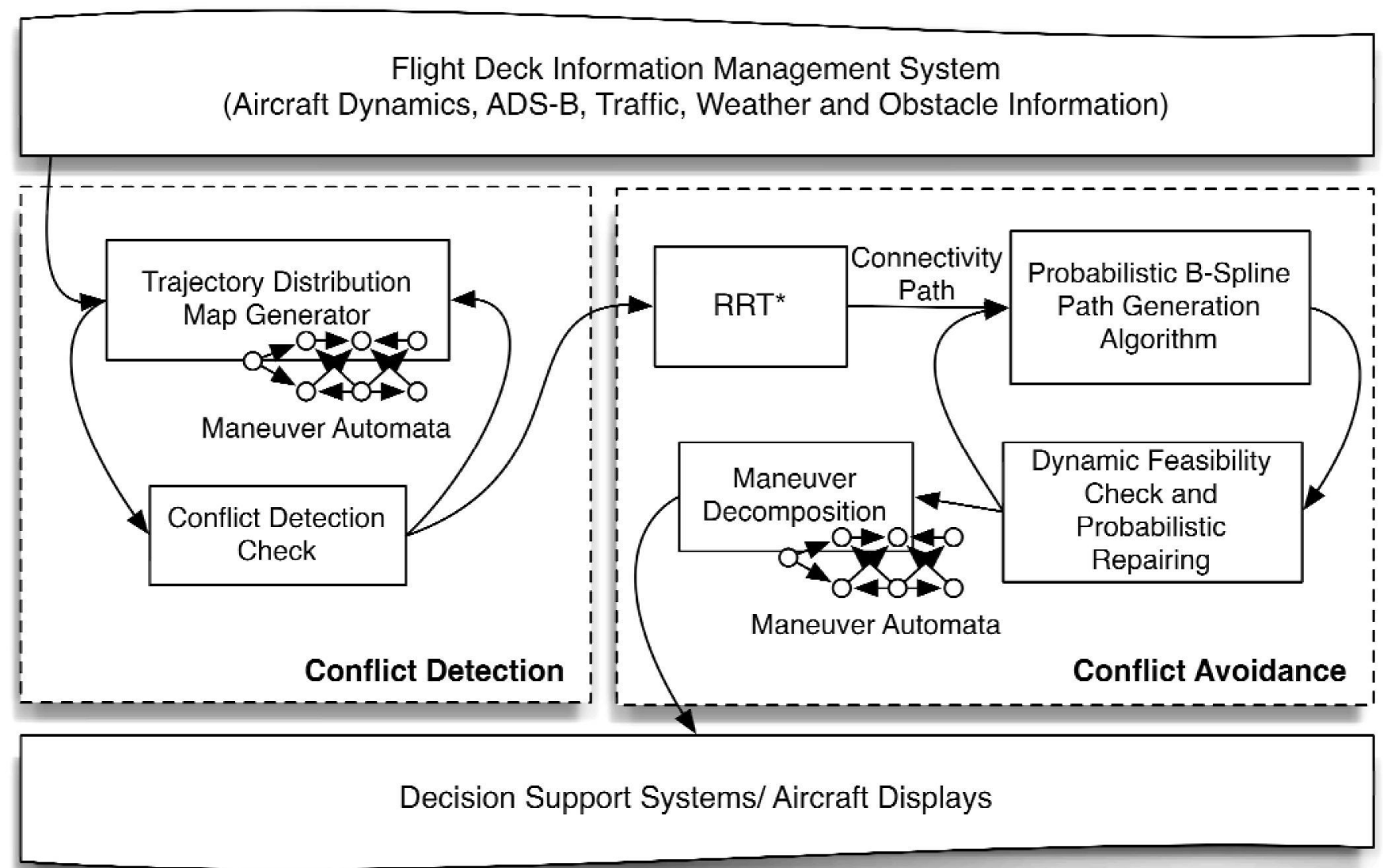

Figure 4. Air Conflict Detection and Resolution Algorithm Architecture 
In addition, visual transformation and presentation of these maps, as seen in Figure 3, AUTOFLY-Aid will provide a natural way of representing the pilot with potential risks and other hazard factors to be encountered in predefined time windows.

\section{Real-Time 4D Trajectory Planning for Pilot Decision Support}

4D trajectory planning methodology hinges on solving relaxed forms of the detected collision avoidance problem and then gradually refining the problem using the flight tracks of approximate solutions. The algorithm envisioned is to use the TCAS solution as the initial solution and further improve on it. In our earlier work [17-20], we observed that before the major feasible path planning phase, defining the geometrical obstacle free path and tractable way points significantly accelerates the searching ability and decreases the total computational time of planner. This approach is considered to be implemented, is into two layers. In the first layer, Trajectory Planning Layer, the algorithm rapidly explores the complex environment with an enhanced Rapidly Exploring Random Tree (RRT $\left.{ }^{*}\right)$ algorithm using its well quick spreading ability. Through these trees, obstacle-free paths can be obtained rapidly. RRT* method [20] is a novel algorithm, which includes asymptotic optimality property while maintaining a tree structure of the RRT algorithm, and were shown to have no significant overhead when compared to the many other randomized sampling algorithms in terms of asymptotic computational complexity. AUTOFLYAid aims to further improve the RRT* method by a) further expanding the methodology to search within inherently stochastic 4D distribution maps in which each path search carries uncertainty measures and by b) parallelizing the search process by additional seeds as to obtain simultaneous possible "avoid" routes across uncertainty boundaries.

In the second layer, obstacle/collision free paths are connected with dynamic B-Spline curves. The approximation can be further verified for collision and dynamic feasibility by computing the first and second derivatives of the spline. These derivatives correspond to the instantaneous velocity and acceleration on the flight path. If the generated curve is not feasible, probabilistic repairing can be achieved by randomized waypoint (control point) placement on the b-spline curves iteratively and then the unit flight time is expanded to limit the acceleration within controllable regime. Since B-Spline curves have local support property, these repairing processes can be made on local path segments of interest without affecting the whole shape of the generated path. After obtaining flight path with velocity history from trajectory planning layer, segment identification readily decomposes the flight path into a sequence of maneuver modes and its parameters. Mode-Based Maneuver Automaton [17,18] implements this decomposition while ensuring transition rules for dynamic feasibility. AUTOFLY-Aid will further study the probabilistic repair of the curves, different curve types and its effects and applicability for dynamically feasible 4D trajectory generation. Generated solution of the method for potential air collision scenario is demonstrated in Figure 3.

AUTOFLY-Aid, focuses on this topic, is envisioned to provide a real-time 4D Trajectory Planning algorithm that can operate across an uncertain trajectory distribution map. In addition the methods developed will assess risks with time, distance and probabilistic measures. With the integration of all these elements, the alternative solutions generated by the composite system will present the pilot with not only alternative and flyable de-conflict trajectories, but also with quantified and visual understanding of collision risks in terms of time and directions. The synthetic vision and reality augmentation, which provides assistance to the flight crew during flight, is illustrated in Figure 5.

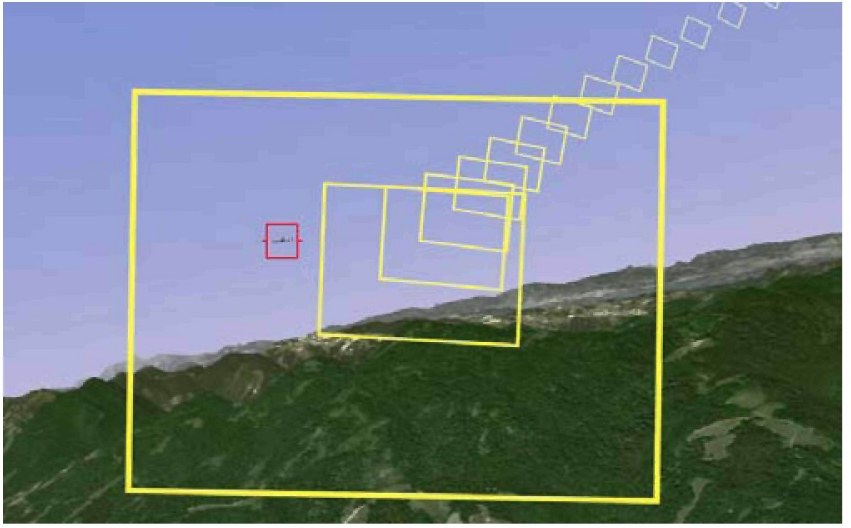

Figure 5. Tunnel-in-the-Sky Synthetic Visualization of the Automatically Generated 4D Trajectories to the Flight Crew 


\section{AUTOFLY-Aid Next Generation Flight Deck Simulator}

AUTOFLY - Aid focuses on dynamic 4D trajectory management using the on - board avionics and the NextGen/SESAR enhanced flight deck situational awareness, specifically coming from SWIM network implementation and from specific devices such as enhanced ADS - B transponders. The automation support system will be demonstrated on a B737-NG Flight Deck Simulator (Figure 1) to provide the pilots with active and visual 4D trajectory management decision support.

Augmented situational awareness will be represented through console avionics displays and head - up displays in real - time. The HUD/AR to be implemented and to be tested includes a) standard pilot-centered HUD, b) enhanced pilot-centered Augmented Reality Goggles c) flight-deck centered Augmented Reality Screen Overlay Projection.

Augmented decision aiding implementations is envisioned to include new touch screen and haptic input devices and switches for moving through HUD and Augmented Reality Display pages and switching/choosing between alternative trajectories represented as tunnels-in-the-sky illustrations.

The composite real-time decision support is to provide the flight crew with quantified and visual understanding of collision risks in terms of time and directions and countermeasures. It will also provide autonomous conflict resolution as a pilot-select autopilot mode. These enhanced functions and the associated in-cockpit avionics within Flight Deck Simulator are illustrated in Figure 6.

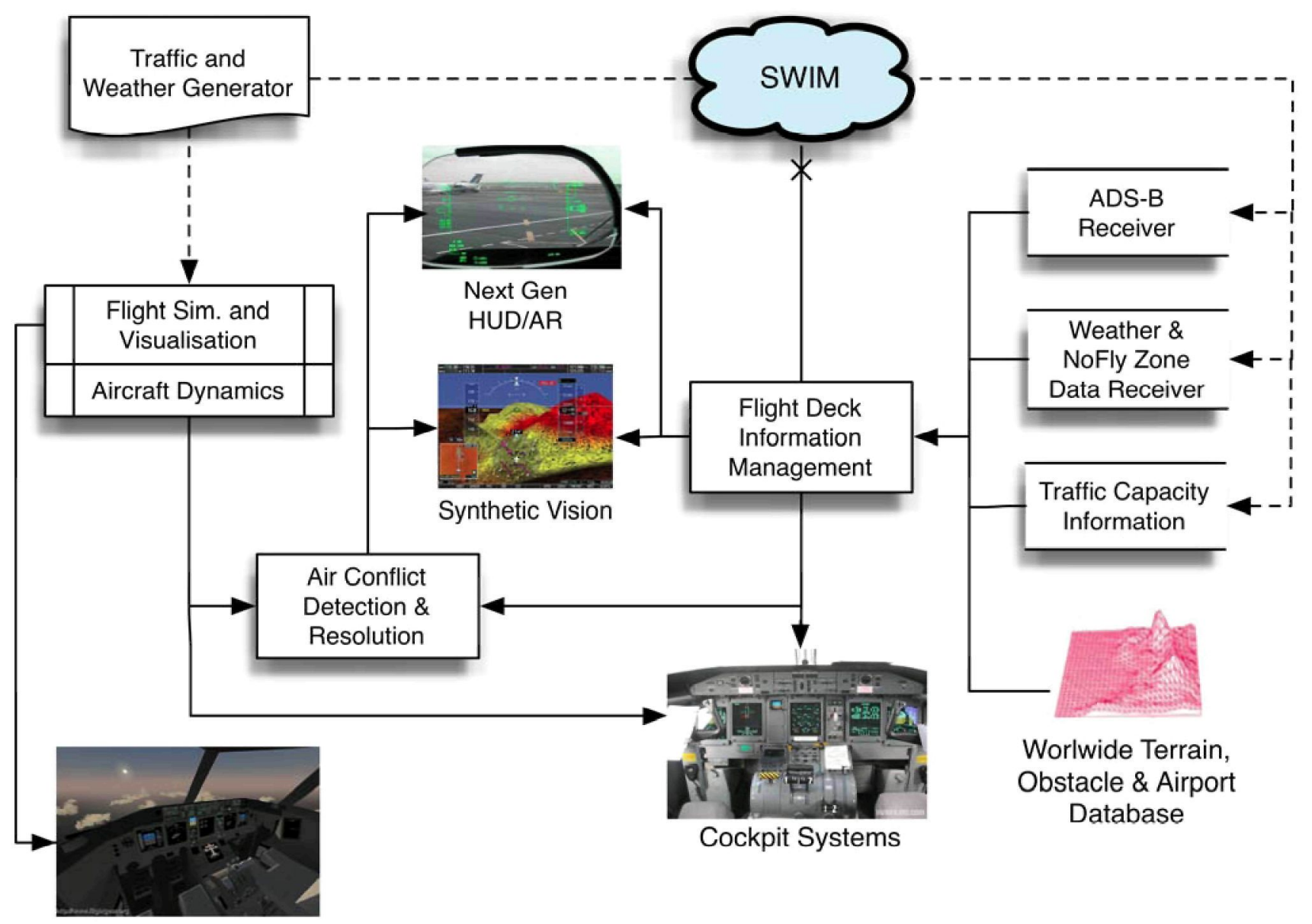

Flight Screen

Figure 6. Next Generation Flight Deck Architecture of AutoFly-Aid Project with Advanced Pilot Decision Support Systems 
In the functional diagram, the Traffic and Weather Generator is used as a scenario builder. Flight Simulator Software visualizes these generated scenarios. The Traffic and Weather Generator (T\&WG) replays both artificially generated scenarios including air congestion and severe weather conditions, or collected live WX and ADS-B data (ITU CAL currently operates a ADS-B radar and EGNOS DCN at Istanbul Ataturk Airport) transmitted from planes operating in East European Airspace. The T\&WG feeds the SWIM cloud in realtime and any external Air Traffic Monitoring System or simulator can be connected to the simulator network by establishing connection to the SWIM. The Flight Deck Information Management (FDIM) system is a local in-cockpit data management system, which gathers all information (such as traffic, capacity, weather, terrain etc.) via communication avionics emulators; parses and broadcasts them to the their clients. For detected potential conflicts (including mid-air and ground), the Air Conflict Detection and Resolution (ACD\&R) system generates dynamic conflict resolution to provide the pilots support with its alternatives and these 4D trajectories and corresponding obstacles are visualized through head-up-display (HUD)/Augmented Reality (AR) and Synthetic Vision display (as seen in Figure 5). The system enables the pilots to switch between alternative dynamically generated solutions via touch-screen, switches and haptic devices. In order to extend situational awareness of the pilot over entire flight operation, the Synthetic Vision display also offers additional pages including long-term, mid-term and short-term threat screens.

\section{Conclusion}

The AUTOFLY-Aid Project aims to develop and demonstrate novel automation support algorithms and tools to the flight crew for flight critical collision avoidance using "dynamic 4D trajectory management". The approach's foundation is based on a hybrid and stochastic dynamic airspace model as seen from the Flight Deck's Perspective. This composite model not only represents the uncertainties associated with sensed and received airspace traffic and intent information but also represents limitations associated with weather, terrain/obstacle and new conflict hazards. The planning layer, using the composite model, generates real-time and dynamically feasible alternative trajectories using an innovative (and provably optimal) stochastic sampling method in which the TCAS RA is the initial solution. These algorithms and tools developed are to be integrated on an automation support system. The automation support system is aimed to improve the primary shortcomings of TCAS, and to aid the pilot through add-on avionics/head-up displays and reality augmentation devices in dynamically evolving collision avoidance scenarios. As a part of the AUTOFLY-Aid Project, the developed automation support system will be demonstrated and tested on an in-house B737 NG FNPT II flight simulator with synthetic vision and reality augmentation.

\section{References}

[1] T.A. Choyce and K.M. Ciaramella, "Test and Evaluation of TCAS II Logic Version 7," Federal Aviation Administration (2000).

[2] J.K. Kuchar and A.C. Drumm, "The Traffic Alert and Collision Avoidance System," Lincoln Laboratory Journal 16(2), 277-296 (2007).

[3] Arinio, T., Decision Criteria for Regulatory Measures on TCAS II Version 7.1, Safety Issue Rectification Extension Plus Project, 2008

[4] German Federal Bureau of Aircraft Accidents Investigation, Investigation Report AX001-1-2/02, BFU Investigation, May, 2004

[5] Single European Sky ATM Research(SESAR) Programme Office, The SESAR Programme; Sustainable Development and Technological Revolution in Air Traffic Management, SESAR factsheet, N 01/2011

[6] Single European Sky ATM Research (SESAR) Programme Office, SESAR Fact Sheet; Business Trajectory/ 4D Trajectory, SESAR Technical Report, N 02/2010, 2010

[7] RTCA Inc. Minimum Aviation System Performance Standards for Automatic Dependent Surveillance Broadcast (ADS-B). Technical Report RTCA/DO-242A, Washington, DC, June 2002.

[8] Olson, W., TCAS Status Update, 2009 CNS/ATM Users Conference, 2009

[9] D. Wing, "A Potentially Useful Role for Airborne Separation in 4D-Trajectory ATM Operations," 5th AIAA Aviation Technology 
Integration and Operations (ATIO) Conference, 2005 .

[10] S. M. Green, K. D. Bilimoria, and M. G. Ballin, "Distributed Air/Ground Traffic Management for En Route Flight Operations." Air Traffic Control Quarterly, Vol.9, No. 4, 2001, pp. 259-285.

[11] K. Krishnamurthy, B. Barmore, and F. Bussink, "Airborne Precision Spacing in Merging Terminal Arrival Routes," 6th USA/Europe Air Traffic Management R\&D Seminar, 2005.

[12] H. A. P. Blom, B. Klein Obbink, and G. I. Bakker, "Safety Risk Simulation of an Airborne Self Separation Concept of Operation," Proceedings of the 7th AIAA Aviation Technology Integration and Operations (ATIO) Conference, AIAA 2007-7729.

[13] R. Barhydt, and P. Kopardekar, "Joint NASA Ames/Langley Experimental Evaluation of Integrated Air/Ground Operations for En Route Free Maneuvering," 6th USA/Europe Air Traffic Management R\&D Seminar, 2005.

[14] MFFP Mediterranean Free Flight Programme Final Report 2005, D821,

[15] J. Kuchar and L. Yang, "A Review of Conflict Detection and Resolution Methods," IEEE Transactions on Intelligent Transportation Systems, vol. 1, no. 4, pp. 179-189, 2000.

[16] Temizel, S., Planning under Uncertainty for Dynamic Collision Avoidance, PhD Thesis, Massachusetts Institute of Technology, 2011

[17] Koyuncu, E., Ure, N. K., and Inalhan, G. Integration of Path/maneuver Planning in Complex
Environments for Agile Maneuvering UCAVs. Int. J. of Intel. and Robotic Systems, 57:143-170, 2010.

[18] Ure, N. K., and Inalhan, G. Feasible Agile Maneuver Identification and Generation Algorithms on Multi Modal Control Framework. AIAA Conference on Guidance Navigation and Control (GNC'09), 2009.

[19] Koyuncu, E., Ure, N. K., and Inalhan, G. A Probabilistic Algorithm for Mode Based Motion Planning of Agile Unmanned Air Vehicles in Complex Environments. International Federation of Automatic Control (IFAC'08) World Congress, 2008.

[20] Koyuncu, E., Karaman S., Frazzoli, E., Inalhan, G. Probabilistic 4D Conflict Detection and Avoidance Strategy in Air Traffic Congestion, Int. Conf. on Application and Theory of Automation in Command and Control Systems, Barcelona, Spain, May 2011.

\section{Acknowledgements}

This work and Emre Koyuncu is supported in part by SESAR WP-E HALA! Research Network $\mathrm{Ph} . \mathrm{D}$. Fellowship. A part of the AUTOFLY-Aid is to be carried in CRIDA, UPM, Eurocontrol and Boeing RTE. The hardware platforms of this project is supported in part by TUBITAK $111 \mathrm{M} 167$ Project Grant.

\section{Integrated Communications Navigation and Surveillance (ICNS) Conference}

April 24-26, 2012 